\title{
Field-aligned current associated with low-latitude plasma blobs as observed by the CHAMP satellite
}

\author{
J. Park ${ }^{1,2}$, H. Lühr ${ }^{1}$, C. Stolle ${ }^{1}$, M. Rother ${ }^{1}$, K. W. Min ${ }^{2}$, and I. Michaelis ${ }^{1}$ \\ ${ }^{1}$ Helmholtz Center Potsdam (GFZ), German Research Center for Geosciences, Telegrafenberg, 14473 Potsdam, Germany \\ ${ }^{2}$ Department of Physics, Korea Advanced Institute of Science and Technology, Korea
}

Received: 14 December 2009 - Revised: 17 February 2010 - Accepted: 21 February 2010 - Published: 2 March 2010

\begin{abstract}
Here we give two examples of low-latitude plasma blobs accompanied by linearly polarized perpendicular magnetic deflections which imply that associated field-aligned currents (FACs) have a 2-D sheet structure located at the blob walls. The estimated FAC density is of the order of $0.1 \mu \mathrm{A} / \mathrm{m}^{2}$. The direction of magnetic deflections points westward of the magnetic meridian and there is a linear correlation between perpendicular and parallel variations. All these properties are similar to those of equatorial plasma bubbles (EPBs). According to CHAMP observations from August 2000 to July 2004, blobs show except for these two good examples no clear signatures of 2-D FAC sheets at the walls. Generally, perpendicular magnetic deflections inside blobs are weaker than inside EPBs on average. Our results are consistent with existing theories: if a blob exists, (1) a significant part of EPB FAC will be closed through it, exhibiting similar perpendicular magnetic deflection inside EPBs and blobs, (2) the FAC closure through blobs leads to smaller perpendicular magnetic deflection at its poleward/downward side, and (3) superposition of different FAC elements might result in a complex magnetic signature around blobs.
\end{abstract}

Keywords. Ionosphere (Electric fields and currents; Equatorial ionosphere; Ionospheric irregularities)

\section{Introduction}

Equatorial plasma bubbles (EPBs) are regions of plasma density depletion at the nighttime equatorial ionosphere, which were first reported by Booker and Wells (1938). EPBs originate from generalized Rayleigh-Taylor instability at the bottomside ionosphere (e.g. Sultan, 1996), and their occurrence probability depends on longitude and season (e.g. Tsunoda,

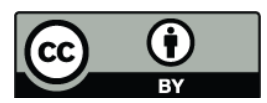

Correspondence to: J. Park

(park@gfz-potsdam.de)
1985; Kil and Heelis, 1998; Huang et al., 2001; Park et al., 2005; Stolle et al., 2006). On the other hand, lowlatitude plasma blobs are regions of abrupt plasma density enhancement observed in the low-latitude ionosphere (poleward of latitudes where EPBs generally occur). They were first reported by Oya et al. (1986) using Hinotori satellite data from $600 \mathrm{~km}$ altitude. Watanabe and Oya (1986) investigated the statistical distribution of blobs encountered by the same satellite. Blobs frequently occurred (1) during solstices, (2) in the winter hemisphere, (3) at postmidnight hours, and (4) near longitudes of EPB occurrence peaks (see their Fig. 10). It was speculated that trans-equatorial neutral wind might play a role in blob generation. Later, Le et al. (2003) reported blob observations using ROCSAT-1 (at $600 \mathrm{~km}$ altitude) and DMSP satellites (at $840 \mathrm{~km}$ altitude). In general, blobs and EPBs were observed near-simultaneously, both drift upward, and their density fluctuation spectra were similar in the scale range between $100 \mathrm{~m}$ and $10 \mathrm{~km}$. Park et al. (2008a) investigated the statistical distribution of blobs using KOMPSAT-1 and DMSP F15. The blob occurrence was higher in the winter hemisphere at both altitudes, corroborating the result of Watanabe and Oya (1986). It was also shown that blob latitudes are higher when the yearly solar activity is higher, implying a close relationship between EIA and blobs. Using magnetic field data from the CHAMP satellite (at about $400 \mathrm{~km}$ altitude) Park et al. (2008b) reported that blobs show reduced magnetic field strength as well as magnetic deflections associated with field-aligned current (FAC). At CHAMP altitude blobs also occurred predominantly in the winter hemisphere.

It is well-known that FACs flow along EPB walls (Aggson et al., 1992; Bhattacharyya and Burke, 2000; Stolle et al., 2006; Pottelette et al., 2007; Park et al., 2009). Aggson et al. (1992) observed for the first time magnetic fluctuations perpendicular to the ambient magnetic field (hereafter, "perpendicular magnetic deflections"), which indicate the existence of FACs. It was suggested that FACs originate from

Published by Copernicus Publications on behalf of the European Geosciences Union. 
the divergence of ambient zonal currents at EPB walls, and they close at the poleward bottomside ionosphere (see Fig. 7 in Aggson et al., 1992). According to this model, FACs generate inward (outward) magnetic deflection in the Northern (Southern) Hemisphere. Bhattacharyya and Burke (2000) extended the idea and argued that FACs are closed not only at the bottomside but also partly in the topside ionosphere by polarization currents driven by time-varying E-fields. Stolle et al. (2006) examined inward/outward directions of perpendicular magnetic deflections observed by CHAMP. However, a significant fraction of examples were inconsistent with the model suggested by Aggson et al. (1992) and Bhattacharyya and Burke (2000). Park et al. (2009) showed that the model is consistent with CHAMP observations only before $\sim 22: 00$ LT. They argued that the assumption of an eastward E-field (used in Aggson et al., 1992; Bhattacharyya and Burke, 2000, and Stolle et al., 2006) might not be valid after $\sim 22: 00$ LT. It was also found that the perpendicular magnetic deflections are not purely inward/outward as suggested by Aggson et al. (1992), but tilted westward by $\sim 40^{\circ}$, which is consistent with the known EPB geometries (Mendillo and Tyler, 1983; Mukherjee, 2003; Makela and Kelley, 2003). Using Ampère's law Park et al. (2009) estimated the FAC density to be of the order of $0.1 \mu \mathrm{A} / \mathrm{m}^{2}$, which is consistent with the result of Pottelette et al. (2007) using E- and B-field measurements.

Although Park et al. (2008b) addressed already the magnetic signatures of blobs, the focus of that study was put on the decrease in magnetic field strength and the maintenance of pressure balance in the region of enhanced plasma density. The perpendicular magnetic deflections (FAC signatures) were not discussed in detail, such as the polarization, amplitude, or associated FAC density. In this study we will address the three properties given above, and interpret the result in the context of the FAC closure mechanism around EPBs. In Sect. 2 we briefly describe the instrumentation. The observational results are given in Sect. 3, and we discuss them in Sect. 4. Finally, the new findings are summarized in Sect. 5.

\section{Instrumentation}

CHAMP (Challenging Mini-satellite Payload) was launched in 2000 into a near-polar (inclination angle: $87.3^{\circ}$ ) circular orbit at an altitude of $450 \mathrm{~km}$. It has a flux-gate magnetometer (FGM) and an Overhauser magnetometer (OVM) to measure geomagnetic field precisely. The FGM conducts 50 measurements per second. The raw data are processed by decimation and cross-calibration to give $1 \mathrm{~Hz}$ level 2 data in geographic coordinate system. A Planar Langmuir Probe (PLP) measures the plasma density every $15 \mathrm{~s}$. Unfortunately, the Digital Ion Drift Meter (DIDM) determining ion density, drift, and temperature was badly degraded during the launch, and we can only get relative (uncali- brated) ion density at a $1 \mathrm{~Hz}$ rate. As of August 2009 all the instruments mentioned above are still in operation at the altitude of $\sim 300 \mathrm{~km}$. All the data are available at (http://isdc.gfz-potsdam.de/index.php).

The Potsdam Magnetic Model of the Earth, Version 4 (POMME4) is an empirical magnetic field model based on CHAMP observations (http://www.gfz-potsdam. de/magmodels/POMME4). With the input of time, location, interplanetary magnetic field and ring current strength, the model outputs a magnetic field vector in geographic coordinate system. For the $1 \mathrm{~Hz}$ FGM data the "residual field" is defined as the difference between the FGM data and the POMME4 output (mean field) in a geographic coordinate system. The residual field is then transformed into the meanfield aligned (MFA) coordinate system defined as follows: "z" axis (labeled "parallel") is parallel to the mean field, "y" axis ("zonal") is perpendicular to the magnetic meridian (positive eastward), " $\mathrm{x}$ " axis ("meridional") completes the triad (positive outward).

Generally, the $50 \mathrm{~Hz}$ FGM data cannot be transformed easily into a geographic coordinate system because the required information on satellite attitude has a $1 \mathrm{~Hz}$ temporal resolution. The $50 \mathrm{~Hz}$ FGM data are typically stored in the sensor coordinate system. Therefore, we first approximate the mean field vector in the sensor frame by the low-pass filtered components of the measurements. A Savitzky-Golay filter (order 3, window size $90 \mathrm{~s}$ ) is used for it. The mean field estimated in this way is used for direct transformation of the high rate magnetic field readings into the MFA coordinate system.

\section{Results}

Figure 1 shows an example of a plasma blob observed by CHAMP. Panels $(\mathrm{a}-\mathrm{c})$ show the three field components at $1 \mathrm{~Hz}$ in MFA coordinates, panel (d) presents the relative plasma density measured by DIDM, and panel (e) the absolute plasma density obtained by PLP. The geomagnetic activity was very low on the day (Dst $\geq-7 \mathrm{nT})$. In the region marked by a red box we can find a blob: the plasma density is enhanced as shown in panels (d) and (e). The magnetic field strength decreases (panel c), and the perpendicular magnetic deflection is outward (panel a) and westward (panel b). Panels (f-h) are obtained from $50 \mathrm{~Hz}$ FGM data. Panel (f) contains a hodogram of the zonal and meridional residual fields. We can find that the perpendicular magnetic deflection is linearly polarized in a direction tilted westward from the magnetic meridian. The red line is the linear regression (hereafter, "direction of maximum variance") obtained by the total least square, and the angle between the regression line and $\mathrm{x}$-axis (hereafter, "polarization angle") is $104^{\circ}$. In panel $(\mathrm{g})$ the black curve represent the perpendicular magnetic deflection along the direction of maximum variance (hereafter, "maximum variance component") and the 

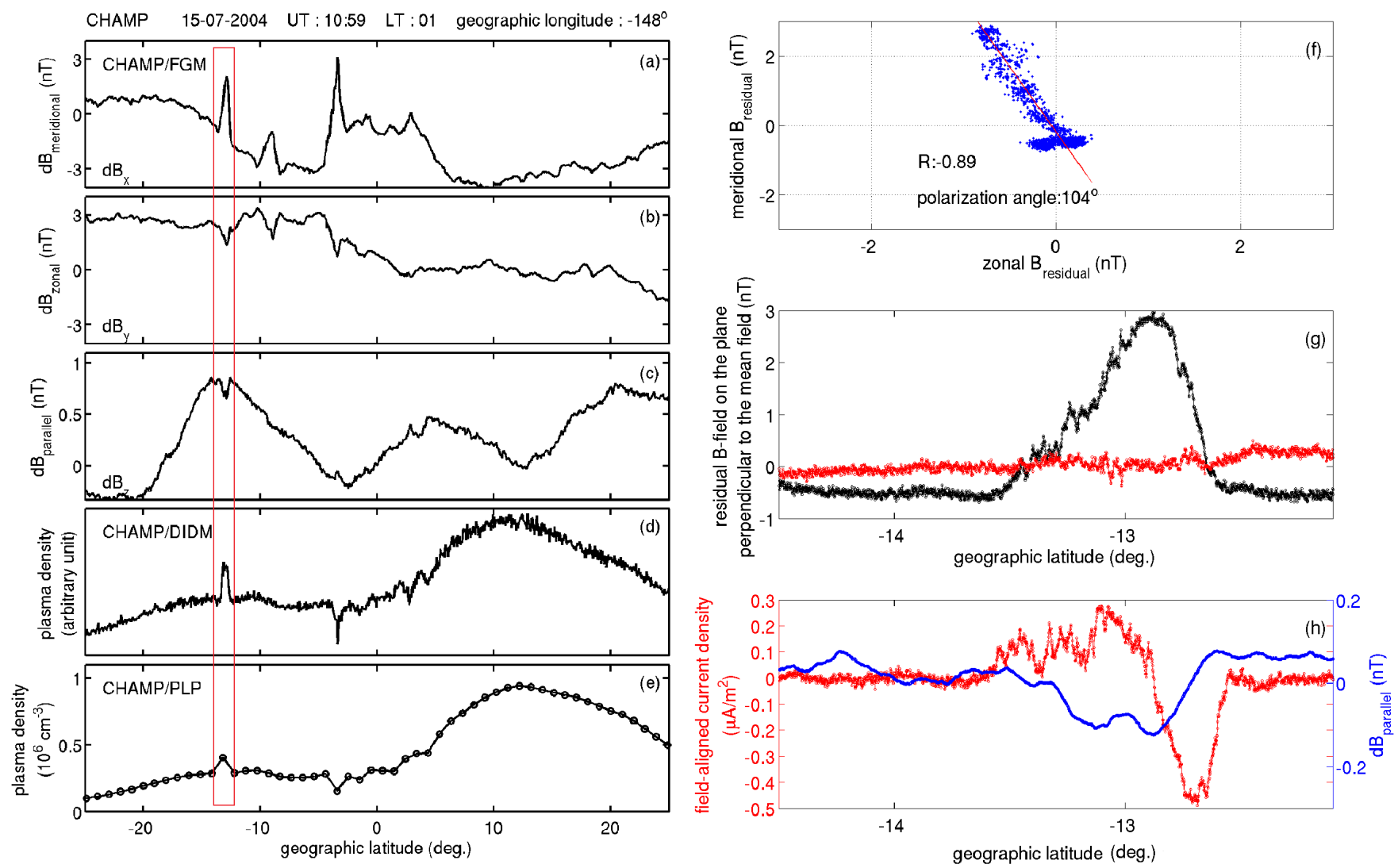

Fig. 1. A blob example observed by CHAMP: (a-c) the three components of the residual field in MFA coordinates, (d) relative plasma density measured by DIDM, (e) absolute plasma density derived from PLP, (f) hodogram of the zonal and meridional residual fields, (g) magnetic deflections along the direction of maximum variance (black curve), and minimum variance (red curve), (h) FAC density (red dots) and parallel component of the residual field (blue curve).

red curve that of minimum variance axis (hereafter, "minimum variance component"). As the maximum variance component is much larger than the minimum variance component, FAC density can be estimated by Ampère's law from a single satellite (Park et al., 2009):

$j_{z}=-\frac{1}{\mu_{0}} \frac{\Delta B_{\perp}}{\Delta s}$

where $j_{z}$ denotes FAC density, $\mu_{0}$ the permeability of free space, $s$ the distance along the minimum variance direction, $B_{\perp}$ the maximum variance component, and $\Delta$ the difference between adjacent values. The resulting FAC density is filtered by a low-pass Savitzky-Golay filter (order 3, window size $3.62 \mathrm{~s}$ ), and given in panel (h) by red dots. Additionally, the blue curve in panel (h) represents the residual field of the parallel component, which is decreased within the blob, as seen in panel (c). The FAC density is of the order of $0.5 \mu \mathrm{A} / \mathrm{m}^{2}$. Currents flow downward at the equatorward (rightside) edge. On the poleward (leftside) side, which exhibits less steep gradient and more sub-structures, FACs generally flow upward.
Park et al. (2008b) identified 52 blobs from CHAMP observations between August 2000 and July 2004. For each of the 52 blobs we evaluated the correlation coefficients between the zonal and meridional components. If the correlation coefficient is above 0.6 , we evaluate the maximum variance component, as shown in Fig. $1 \mathrm{~g}$. Only for 2 blobs both correlation coefficients (1) between the zonal and meridional and (2) between parallel and maximum variance components are above 0.6. One of them is shown in Fig. 1. For the other one (near 04:10 UT on 18 January 2002, shown in Fig. 1 of Park et al., 2008b), the polarization angle is $150^{\circ}$ (i.e. westward tilt) and the peak FAC density is of the order of $0.1 \mu \mathrm{A} / \mathrm{m}^{2}$.

Figure 2 shows another blob example observed by CHAMP in the same format as that of Fig. 1. In the region marked by a red box we find a blob. While the parallel component decreases inside the blob (panel c), associated perpendicular magnetic deflections are inconspicuous (panels a and b). In contrast, EPBs observed in this orbit, e.g. the event around $15^{\circ}$ geographic latitude, show clear perpendicular magnetic deflections. The EPB around $-13^{\circ}$ and the blob around $+28^{\circ}$ geographic latitude are near $\mp 20^{\circ}$ geomagnetic 


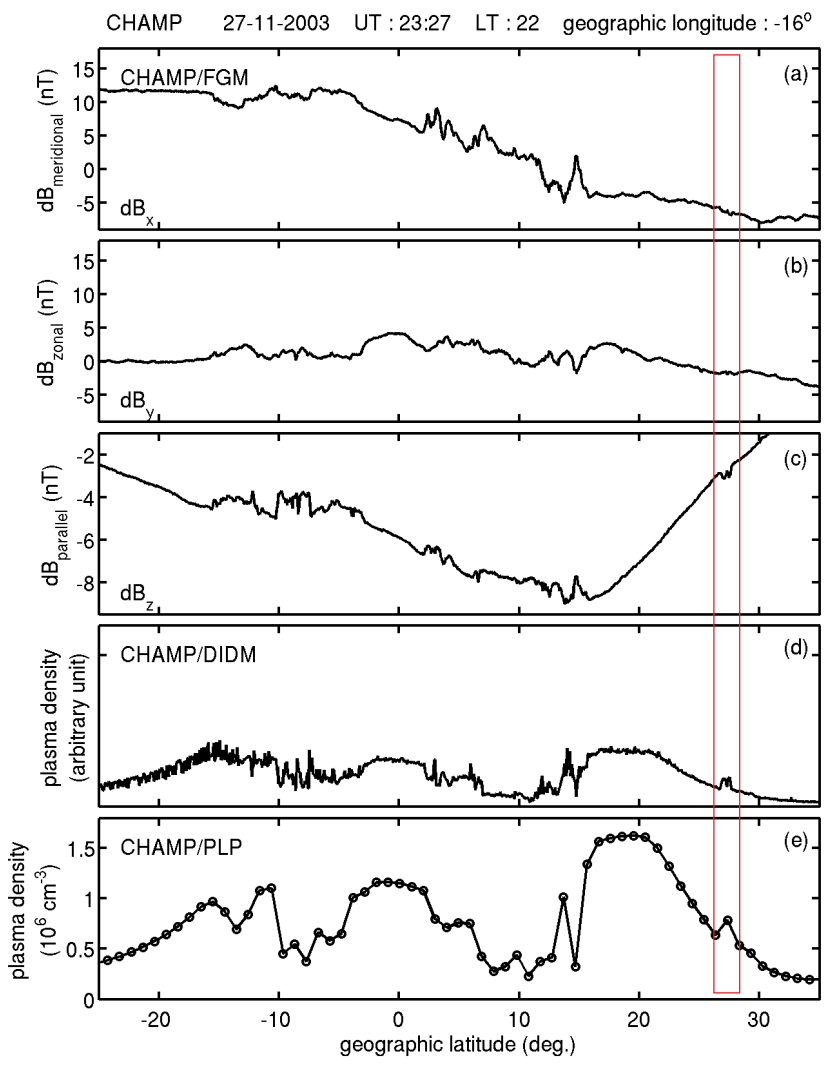

Fig. 2. Another blob example in the same format (with different scales) as in Fig. 1.

latitude, respectively. However, the EPB exhibits a much clearer signature in the perpendicular components than the blob, suggesting significant FACs associated with the EPB. Note that Figs. 1 and 2 have different scales for convenience, but the arguments given in this paragraph remain valid even when the figures are compared on the same scale.

For most blobs observed by CHAMP, perpendicular magnetic deflections were not as conspicuous as for the one in Fig. 1. For each of the 52 blobs we filtered/rectified the $1 \mathrm{~Hz}$ FGM data with a high-pass filter $\left(T_{c}=10 \mathrm{~s}\right)$ and recorded the maximum amplitude of zonal/meridional deflection. The mean of the maximum amplitudes is $0.37 \pm 0.31$ (nT). We applied the same procedure to EPBs, and the result is $0.84 \pm$ 0.90 (nT).

\section{Discussion}

New findings in the previous section are as follows. First, CHAMP observed two blobs in which perpendicular magnetic deflections are linearly polarized and their maximum variance component is well correlated with the parallel component. The polarization vector was tilted westward from the magnetic meridian. The magnetic signatures imply that associated FACs are 2-D sheets located at the blob walls and tilted westward from vertical: similar to the EPB FAC configuration reported in Park et al. (2009). Making use of the linear polarization we estimated blob FAC densities to be of the order of $0.1 \mu \mathrm{A} / \mathrm{m}^{2}$. Second, for the other 50 blobs from August 2000 to July 2004, the magnetic signature of "2-D FAC sheets at the walls" was not as evident. Third, perpendicular magnetic deflections associated with blobs were generally smaller than those associated with EPBs.

Before discussing the global context of blob FACs, the observed magnetic signatures of EPBs and blobs need to be compared in some detail. The blob shown in Fig. 1 is located in the Pacific sector, where EPB activity is high around June solstice (e.g. Kil and Heelis, 1998; Huang et al., 2001; Park et al., 2005; Stolle et al., 2006), and in the Southern winter Hemisphere where blobs are frequently observed (e.g. Watanabe and Oya, 1986; Park et al., 2008b). This event is accompanied by EPBs between $\pm 3^{\circ}$ GLAT showing magnetic field deflections outward and westward. Magnetic deflections inside the blob have the same polarization (outward/westward). For the other example on 18 January 2002 (00:00 LT), the blob appears in the Atlantic sector $\left(-58^{\circ}\right.$ GLON) and in the Northern winter Hemisphere with outward/westward magnetic deflection. EPBs on the same pass (see Fig. 1 of Park et al., 2008b) also show outward/westward magnetic deflection in both hemispheres. Namely, the magnetic signatures of the two blobs are polarized in the same direction as the accompanying EPBs in both hemispheres. According to Park et al. (2009) meridional magnetic deflections inside EPBs show hemispheric antisymmetry before 22:00 LT, but the asymmetry disappears afterwards. This is consistent with our two blob events which occurred past midnight.

Let us interpret these results in the context of the lowlatitude ionospheric FAC circuit. Stolle et al. (2006) argued that FACs are the source of zonal/meridional magnetic deflection observed by CHAMP/FGM inside EPBs. There is a consensus that those FACs are (1) generated by diverted zonal background currents at field-aligned EPB walls and (2) closed at conjugate conducting layers at lower altitude (Aggson et al., 1992; Bhattacharyya and Burke, 2000; Stolle et al., 2006). There is another consensus that blobs are nearconjugate to EPBs: no matter whether (1) the enhanced Efield is mapped along geomagnetic field lines to unperturbed EIA latitudes and lifts up dense plasma from the bottomside (Le et al., 2003; Pimenta et al., 2004; Yokoyama et al., 2007) or (2) the super-fountain effect accumulates plasma at the poleward EPB boundaries (Park et al., 2003; Martinis et al., 2009; Krall et al., 2009). A natural consequence of these two consensi is: a blob, if any, is a part of the "conjugate conducting layer" of an EPB and closes a portion of its FAC. Moreover, the portion is expected to be significant because of higher cross-field conductivity in blobs than in the ambient.

The blob magnetic signatures shown in Figs. 1 and 2 are consistent with the above argument. As blobs can be the closure paths of EPB FACs, associated perpendicular magnetic 
(a) symmetric B-field deflection (current closure mainly in one hemisphere)
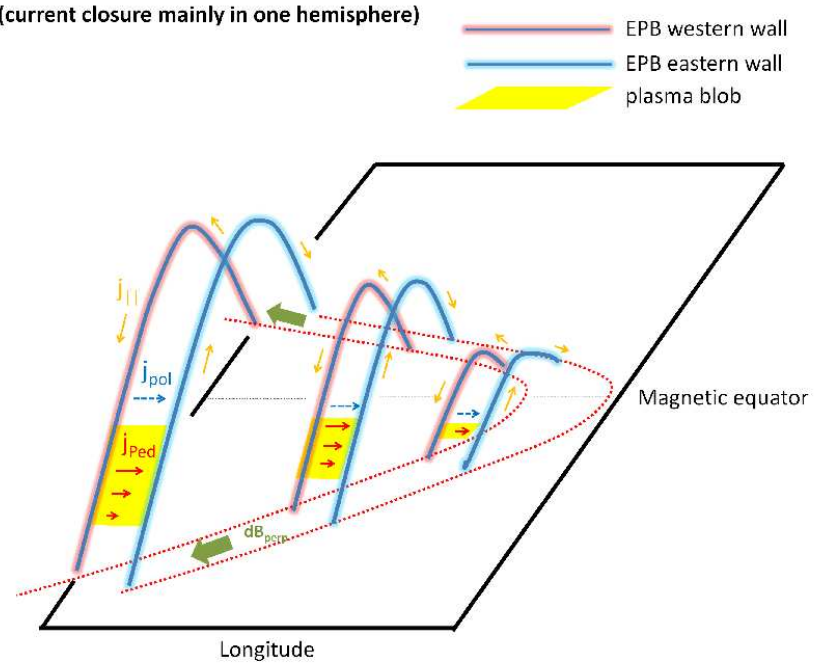

(b) anti-symmetric B-field deflection (current closure in both hemispheres)

EPB western wall EPB eastern wall

Fig. 3. A schematic diagram of FAC circuits associated with (a) EPBs and blobs (b) EPBs only (adapted version of Fig. 8 in Park et al. (2009)). Yellow patches mark locations of plasma blobs.

deflections are similar in both blobs and EPBs: linear polarization and westward tilt. At the topside (equatorward side) of a field-aligned blob structure, perpendicular magnetic deflection would have comparable amplitude to that in EPBs. However, at the bottomside (poleward side), the deflection would be much diminished due to the FAC closure at the topside. Figures 1 and 2 might represent blob encounters at its topside and bottomside, respectively. As perpendicular magnetic deflections were on average smaller in blobs than in EPBs, CHAMP might have been traversing blobs frequently at their bottomside (due to its low orbit altitude). This suggestions will be tested by ESA's upcoming Swarm mission (http://www.esa.int/esaLP/LPswarm.html). It consists of 3 satellites at 2 different altitudes, all with high-precision magnetometers. Swarm can verify whether perpendicular magnetic deflections are really larger at the topside of a blob than at its bottomside conjugate point.

Figure 3 is a schematic diagram of the FAC circuit associated with EPBs and blobs. Recently Kil et al. (2009) and Kil and Paxton (2009) suggested that EPBs have shell structures, based on which Park et al. (2009) described the FAC circuit associated with EPBs (see their Fig. 8b). This idea is further extended in our Fig. 3. Note that the blob in Fig. 3a exists in only one hemisphere, reflecting the hemispheric asymmetry during solstices (Watanabe and Oya, 1986; Park et al., 2008a,b). Let us assume a net eastward ambient current at the topside. First, FACs (orange arrows) are generated at the wall of the EPB due to the divergence of background current. Second, parts of FACs are closed within the EPB by polarization current (blue arrow). Third, as blobs of higher conductivity (yellow area) are located at the high-latitude EPB boundaries, a large part of EPB FACs is closed by the Pedersen current through blobs. Hence, the lower the satellite crosses a blob, the weaker are the observed perpendicular magnetic deflections.

There is one interesting feature to be noted. In Fig. 1 of this paper and of Park et al. (2008b) the polarization senses in the blobs and EPBs are outward/westward in both hemispheres (hemispheric symmetry). Note that both events occurred past midnight. The trend is quite different for the EPB events observed before 22:00 LT, which show hemispheric antisymmetry in magnetic deflections (see Fig. 3b): inward/eastward (outward/westward) in the Northern (Southern) Hemisphere (Park et al., 2009). The hemispherically symmetric polarization implies that the closure of the FACs occurs mainly in one hemisphere. The late (e.g. Martinis et al., 2009) and winter-biased (e.g. Watanabe and Oya, 1986; Park et al., 2008a,b) appearance of solstitial blobs is consistent with those results. During the early hours past sunset, when blobs have not yet been formed, a balanced current closure in both hemispheres is maintained to produce hemispheric antisymmetry in perpendicular magnetic deflections. At later (e.g. post-midnight) hours blobs occur, but generally only in the winter hemisphere so that most FACs are closed there. In that respect blobs can be an important part of the EPB dynamics at later local times.

Let us discuss the significance of the results. Only two blobs out of 52 showed magnetic signatures which imply the existence of 2-D FAC sheets at the walls. 16 blobs among the others have maximum zonal/meridional magnetic deflections less than $0.2 \mathrm{nT}$, which can be considered as a quite faint effect (note that Park et al., 2009, used 0.4 nT to identify significant zonal/meridional deflection within EPBs). These 18 events can be interpreted in the context of EPB/blob FAC circuit described above: the former two (e.g. Fig. 1) are blob topside observations reflecting EPB FAC signatures, 
and the latter 16 (e.g. Fig. 2) are blob bottomsides showing faint signatures. For the remaining 34 blobs the maximum zonal/meridional magnetic deflections are larger than $0.2 \mathrm{nT}$, but the linearly polarized signature of 2-D FAC sheets at the walls is not as evident. These poor examples need to be incorporated into the above-mentioned scheme: blobs can be closure paths of EPB FAC. We note that a blob-associated region detected by CHAMP (e.g. Fig. 2) actually consists of smaller flux tubes, each of which has its own FAC circuit. FACs in each flux tube might have a different amplitude due to different conductivity of the conjugate EPB. Also, vertical tilt of flux tubes might not be the same, leading to different polarization angles. Hence, superposition of such substructures can result in the magnetic signatures of the poor examples. Similar arguments were given for EPBs in Park et al. (2009). Meanwhile, once blob magnetic deflections show clear signatures of 2-D FAC sheets at the walls, (1) the FAC sheet reveals clear westward tilt, and (2) the polarization is the same for all blobs and EPBs on a given pass, implying FAC closure in only one hemisphere (blob hemisphere).

\section{Summary}

From magnetic field deflections inside low-latitude plasma blobs, as observed by the CHAMP/FGM, we found the following:

1. On 15 July 2004 (01:00 LT) and 18 January 2002 (00:00 LT) CHAMP encountered low-latitude plasma blobs in which perpendicular magnetic deflections are linearly polarized (tilted toward west from the magnetic meridian) and their maximum variance component is well correlated with the parallel component. The magnetic signatures imply that the blobs have 2-D FAC sheet structures at the walls and are tilted westward from the magnetic meridian: similarly to EPBs as can be seen in Park et al. (2009). The FAC density estimated from the perpendicular magnetic deflections is of the order of $0.1 \mu \mathrm{A} / \mathrm{m}^{2}$.

2. According to CHAMP observations from August 2000 to July 2004, blobs other than the two good examples do not show conspicuous signatures of 2-D FAC sheets at the walls. Generally, perpendicular magnetic deflections inside blobs are weaker than inside EPBs on average.

3. The results are consistent with the prediction of previous theories: FACs originating from EPBs are partly closed through blobs. According to this perspective, perpendicular magnetic deflections at the blob topside are expected to be stronger and comparable to those of EPBs (Summary 1) while they are much weaker at the bottomside (Summary 2). Superposition of different FAC sub-structures might result in a complex magnetic deflection around blobs.
Acknowledgements. The CHAMP mission is supported by the German Aerospace Center (DLR) in operation and by the Federal Ministry of Education (BMBF), as part of the Geotechnology Programme, in data processing. J. Park thanks H. Kil at JHU/APL for fruitful discussions.

Topical Editor M. Pinnock thanks one anonymous referee for her/his help in evaluating this paper.

\section{References}

Aggson, T. L., Burke, W. J., Maynard, N. C., Hanson, W. B., Anderson, P. C., Slavin, J. A., Hoegy, W. R., and Saba, J. L.: Equatorial bubbles updrifting at supersonic speed, J. Geophys. Res., 97, 8581-8590, 1992.

Bhattacharyya, A. and Burke, W. J.: A transmission line analogy for the development of equatorial ionospheric bubbles, J. Geophys. Res., 105, 24941-24950, 2000.

Booker, H. G. and Wells, H. W.: Scattering of radio waves by the F region of the ionosphere, J. Geophys. Res., 43, 249-256, 1938.

Huang, C. Y., Burke, W. J., Machuzak, J. S., Gentile, L. C., and Sultan, P. J.: DMSP observations of equatorial plasma bubbles in the topside ionosphere near solar maximum, J. Geophys. Res., 106, 8131-8142, 2001.

Kil, H. and Heelis, R. A.: Global distribution of density irregularities in the equatorial ionosphere, J. Geophys. Res., 103, 39693982, 1998.

Kil, H., Heelis, R. A., Paxton, L. J., and Oh, S. J.: Formation of a plasma depletion shell in the equatorial ionosphere, J. Geophys. Res., 114, A11302, doi:10.1029/2009JA014369, 2009.

Kil, H. and Paxton, L. J.: The morphology of the large equatorial plasma depletions observed during the 29-30 October 2003 storm, J. Atmos. Solar-Terr. Phys., submitted, 2009.

Krall, J., Huba, J. D., and Martinis, C. R.: Three-dimensional modeling of equatorial spread $\mathrm{F}$ airglow enhancements, Geophys. Res. Lett., 36, L10103, doi:10.1029/2009GL038441, 2009.

Le, G., Huang, C.-S., Pfaff, R. F., Su, S.-Y., Yeh, H.-C., Heelis, R. A., Rich, F. J., and Hairston, M.: Plasma Density Enhancements Associated with Equatorial Spread F: ROCSAT1 and DMSP observations, J. Geophys. Res., 108, 1318, doi:10.1029/2002JA009592, 2003.

Makela, J. J. and Kelley, M. C.: Field-aligned 777.4-nm composite airglow images of equatorial plasma depletions, Geophys. Res. Lett., 30(8), 1442, doi:10.1029/2003GL017106, 2003.

Martinis, C., Baumgardner, J., Mendillo, M., Su, S.-Y., and Aponte, N.: Brightening of $630.0 \mathrm{~nm}$ equatorial spreadF airglow depletions, J. Geophys. Res., 114, A06318, doi:10.1029/2008JA013931, 2009.

Mendillo, M. and Tyler, A.: Geometry of Depleted Plasma Regions in the Equatorial Ionosphere, J. Geophys. Res., 88(A7), 57785782, 1983.

Mukherjee, G. K.: Studies of the equatorial F-region depletions and dynamics using multiple wavelength nightglow imaging, J. Atmos. Solar-Terr. Phys., 65(3), 379-390, 2003.

Oya, H., Takahashi, T., and Watanabe, S.: Observation of low latitude ionosphere by the impedance probe on board the Hinotori satellite, J. Geomagn. Geoelectr., 38, 111-123, 1986.

Park, J., Min, K. W., Lee, J.-J., Kil, H., Kim, V. P., Kim, H.-J., Lee, E., and Lee, D. Y.: Plasma Blob Events Observed by KOMPSAT-1 and DMSP F15 in the Low Latitude 
Nighttime Upper Ionosphere, Geophys. Res. Lett., 30, 2114, doi:10.1029/2003GL018249, 2003.

Park, J., Min, K. W., Lee, J.-J., Kil, H., Kim, V. P., Kim, H.J., Lee, E., and Lee, D. Y.: Global Distribution of Equatorial Plasma Bubbles in the Premidnight Sector During Solar Maximum as Observed by KOMPSAT-1 and Defense Meteorological Satellite Program F15, J. Geophys. Res., 110, A07308, 10.1029/2004JA010817, 2005.

Park, J., Min, K. W., Kim, V. P., Kil, H., Kim, H. J., Lee, J. J., Lee, E., Kim, S. J., Lee, D. Y., and Hairston, M.: Statistical Description of Low-Latitude Plasma Blobs as Observed by DMSP F15 and KOMPSAT-1, Adv. Space Res., 41(4), 650-654, $2008 \mathrm{a}$.

Park, J., Stolle, C., Lühr, H., Rother, M., Su, S.-Y., Min, K. W., and Lee, J.-J.: Magnetic signatures and conjugate features of low-latitude plasma blobs as observed by the CHAMP satellite, J. Geophys. Res., 113, A09313, doi:10.1029/2008JA013211, 2008b.

Park, J., Lühr, H., Stolle, C., Rother, M., Min, K. W., and Michaelis, I.: The characteristics of field-aligned currents associated with equatorial plasma bubbles as observed by the CHAMP satellite, Ann. Geophys., 27, 2685-2697, 2009, http://www.ann-geophys.net/27/2685/2009/.

Pimenta, A. A., Sahai, Y., Bittencourt, J. A., Abdu, M. A., Takahashi, H., and Taylor, M. J.: Plasma Blobs Observed by Ground-Based Optical and Radio Techniques in the Brazilian Tropical Sector, Geophys. Res. Lett., 31, L12810, doi:10.1029/2004GL020233, 2004.
Pottelette, R., Malingre, M., Berthelier, J. J., Seran, E., and Parrot, M.: Filamentary Alfvénic structures excited at the edges of equatorial plasma bubbles, Ann. Geophys., 25, 2159-2165, 2007, http://www.ann-geophys.net/25/2159/2007/.

Stolle, C., Lühr, H., Rother, M., and Balasis, G.: Magnetic signatures of equatorial spread $\mathrm{F}$, as observed by the CHAMP satellite, J. Geophys. Res., 111, A02304, doi:1029/2005JA011184, 2006.

Sultan, P. J.: Linear theory and modeling of the Rayleigh-Taylor instability leading to the occurrence of equatorial spread F, J. Geophys. Res., 101, 26875-26891, 1996.

Tsunoda, R. T.: Control of the seasonal and longitudinal occurrence of equatorial scintillations by the longitudinal gradient in the integrated E-region Pedersen conductivity, J. Geophys. Res., 90, 447-456, 1985.

Watanabe, S. and Oya, H.: Occurrence Characteristics of Low Latitude Ionospheric Irregularities Observed by Impedance Probe on Board Hinotori Satellite, J. Geomag. Geoelectr., 38, 125-149, 1986.

Yokoyama, T., Su, S.-Y., and Fukao, S.: Plasma blobs and irregularities concurrently observed by ROCSAT-1 and Equatorial Atmosphere Radar, J. Geophys. Res., 112, A05311, doi:10.1029/2006JA012044, 2007. 